\title{
СУЖЕНИЕ ПРОВАЛА В СПЕКТРЕ ПОГЛОЩЕНИЯ ТРЕХУРОВНЕВОЙ СИСТЕМЫ ПРИ ИМПУЛЬСНЫХ ФОТОВЫЖИГАНИИ И ВОЗБУЖДЕНИИ
}

\author{
(Представил В. Хижняков)
}

В [ ${ }^{1}$ ] рассматривалось двухступенчатое фотовыжигание провала в функции неоднородного распределения частот оптических переходов в трехуровневой системе двумя следующими друг за другом импульсами. Целью данной работы является изучение формы и ширины отмеченного провала с помощью стационарного и импульсюго зондирующих излучений. Показано, что в первом случае-с-сежтр-пвухстуненнатөго поглощения можно получить провал шириной меньше $2 \gamma_{1}$ (но больше $\gamma_{1}$ ), а во втором - этот провал, в принципе, может быть сколь угодно узкий, т. е. шириной меньше $\gamma_{1}\left(\gamma_{1}-\right.$ константа энергетической (продольной) релаксации в первом возбужденном состоянии).

Пусть фотовыжиганне происходит следующими когерентными импульсами: первый импульс затухает по экспоненциальному закону (частотное распределение импульса имеет форму лоренциана), а второй является $\delta$-импульсом. В этом случае $\left(T_{1}<T_{2}\right)$ функция, описывающая временное поведение выжигающего поля, следующая:

$g\left(t_{2}, t_{1}\right)=\left\{\begin{array}{l}0, \text { для } t_{2}<T_{1}, \\ \sqrt{\Delta} \exp \left[-i \omega_{0} t_{2}-\Delta\left(t_{2}-T_{1}\right) / 2\right] \delta\left(t_{1}-T_{2}\right), \text { для } t_{2} \geqslant T_{1},\end{array}\right.$

где $T_{1}$ - момент времени прохождения через центр системы максимума первого импульса, $T_{2}$ - момент времени прохождения через центр системы второго импульса, $\omega_{0}$ - частота максимума и $\Delta$ - спектральная ширина первого импульса (полная ширина на половине высоты), $\Delta^{-1}-$ его длительность во времени.

Для описания трехуровневой системы используем простейшую модель, в которой релаксационные процессы в первом возбужденном состоянии 1 и во втором возбужденном состоянии 2 описывают константами энергетической (продольной) релаксации $\gamma_{1}$ и $\gamma_{2}$. Соответствующая корреляционная функция трехуровневой системы следующая:

$$
\begin{gathered}
F\left(t, t_{1}, t^{\prime}{ }_{1}, t_{2}, t_{2}^{\prime}\right)=C \exp \left[-\gamma_{2} t+i \Omega_{12}\left(t_{1}-t_{1}^{\prime}\right)+\gamma_{2}\left(t_{1}+t^{\prime}{ }_{1}\right) / 2+\right. \\
\left.+i \Omega_{01}\left(t_{2}-t^{\prime}{ }_{2}\right)-\gamma_{1}\left(t_{1}+t^{\prime}{ }_{1}-t_{2}-t^{\prime}\right) / 2\right] .
\end{gathered}
$$

Здесь $\Omega_{01}$ и $\Omega_{12}$ - частоты электронных переходов между начальным 0 и промежуточным 1 и между промежуточным 1 и конечным 2 уровнями, C - константа.

В дальнейшем предполагается, что частоты первого импульса находятся в резонансе с переходом $0 \rightarrow 1$, но не с переходом $1 \rightarrow 2$, а частоты второго импульса в резонансе с переходом $1 \rightarrow 2$, но не с переходом $0 \rightarrow 1$. 
После фотовыжигания функция неоднородного распределения энергии оптического перехода $0 \rightarrow 1\left[^{2}\right]$ будет иметь вид $\left[{ }^{1}\right]:$

$$
\mathrm{Q}\left(\Omega_{01}\right)=\mathrm{Q}_{0}\left(\Omega_{01}\right)\left[1-x e^{-\gamma_{1} T} \int_{0}^{T} \int d z d z^{\prime} e^{i x\left(z-z^{\prime}\right)} e^{a\left(z+z^{\prime}\right) / 2}\right],
$$

где $x=\alpha C \Delta / \gamma_{2}, T=T_{2}-T_{1}, a=\gamma_{1}-\Delta, x=\Omega_{01}-\omega_{0}, \quad \alpha-$ вероятность превращения возбужденной на уровень 2 системы, в результате чего она перестает поглощать на прежней частоте $\Omega_{01}$ перехода $0 \rightarrow 1$. Или после интегрирования:

$$
\varrho\left(\Omega_{01}\right)=\varrho_{\theta}\left(\Omega_{01}\right)\left[1-x e^{-\Delta T}\left(1+e^{-a T}-2 e^{-a T / 2} \cos x T\right) /\left(x^{2}+a^{2} / 4\right)\right] .
$$

$\varrho_{0}\left(\Omega_{01}\right)$ - первоначальная функция неоднородного распределения.

Из формулы (4) видим, что получаемый провал в функции неоднородного распределения энергии оптического перехода $0 \rightarrow 1$ имеет осцилляционную форму, причем ширина центрального максимума зависит не от суммы константы энергетической релаксации уровня $1 \gamma_{1}$ и спектральной ширины выжигающего света $\Delta$ (как это имеет место при стационарном выжигании), а от их разницы. Таким образом, наблюдается эффект компенсации спектральной ширины первого выжигающего импульса и ширины, обусловленной затуханием возбужденного уровня 1. Этот эффект аналогичен эффекту компенсации в зависящем от времени спектре резонансного вторичного свечения $\left[{ }^{3}\right]$. Формула (4) аналогична формуле, описывающей зависящий от времени спектр люминесценции (или поглощения) [3]. Провал монотонно сужается с ростом промежутка времени $T$ между выжигающими импульсами. Его предельная ширина определяется разницей параметров $\left|\gamma_{1}-\Delta\right|$. Таким образом, провал в функции распределения частот перехода $0 \rightarrow 1$ может быть уже как $\gamma_{1}$, так и $\Delta$. Когда $\gamma_{1}$ и $\Delta$ скомпенсированы полностью, т. е. $\gamma_{1}=\Delta$, то ширина провала пропорциональна $T^{-1}$.

Рассмотрим, каким образом отмеченный провал в функции $\varrho\left(\Omega_{01}\right)$ проявляется в спектре двухступенчатого поглощения.

1. Рассмотрим стационарное двухступенчатое поглощение. Пусть первый этап двухступенчатого поглощения проводится монохроматической волной с частотой $\omega_{0}{ }^{\prime}$, которая находится в полосе переходов $0 \rightarrow 1$, но не в полосе переходов $1 \rightarrow 2$, а второй этап $(1 \rightarrow 2)-$ широким спектром частот. Соответствующие функции спектрального распределения $\left|f_{1}\left(\omega_{1}\right)\right|^{2}=\delta\left(\omega_{1}-\omega_{0}^{\prime}\right)$ и $\left|f_{2}\left(\omega_{2}\right)\right|^{2}=G=$ const.

Двухступенчатое поглощение $I_{s}(y)$ пропорционально вероятности $W_{s}(y)$ найти систему в состоянии 2

$$
\begin{aligned}
& I_{s}(y) \sim W_{s}(y)=\iint d \omega_{\omega_{1}} d \omega_{2}\left|f_{1}\left(\omega_{1}\right)\right|^{2}\left|f_{2}\left(\omega_{2}\right)\right|^{2} \int d \Omega_{01}\left(\Omega_{01}\right) \times \\
& \times \int_{-\infty}^{t} d t_{1} d t^{\prime}{ }_{1} e^{-i \omega_{2}\left(t_{1}-t_{1}^{\prime}\right)} \int_{-\infty}^{t_{1}} d t_{2} \int_{-\infty}^{t_{1}^{\prime}} d t^{\prime}{ }_{2} F\left(t, t_{1}, t_{1}^{\prime}, t_{2}, t^{\prime}{ }_{2}\right) e^{-i \omega_{1}\left(t_{2}-t^{\prime}{ }_{2}\right) .}
\end{aligned}
$$

Используя для $\mathrm{Q}\left(\Omega_{01}\right)$ формулу (3) (предполагая, что $\mathrm{Q}_{0}\left(\Omega_{01}\right)=$ $=\varrho_{0}=$ const), получаем, интегрируя формулу (5)

$$
\begin{aligned}
& I_{s}(y) \sim \frac{(2 \pi)^{2} \mathrm{Q}_{0} G C}{\gamma_{1} \gamma_{2}}\left\{1-x e^{-\Delta T}\left[\frac{\left(a+\gamma_{1}\right)}{a \beta_{1}}-\frac{\left(\gamma_{1}-a\right)}{a \beta_{2}} e^{-a T}-\right.\right. \\
& \left.\left.-\frac{2}{\beta_{1} \beta_{2}} e^{-\left(a+\gamma_{1}\right) T / 2}\left(\left(y^{2}-\frac{\left(\gamma_{1}^{2}-a^{2}\right)}{4}\right) \cos y \mathrm{~T}+\gamma_{1} y \sin y \mathrm{~T}\right)\right]\right\},
\end{aligned}
$$




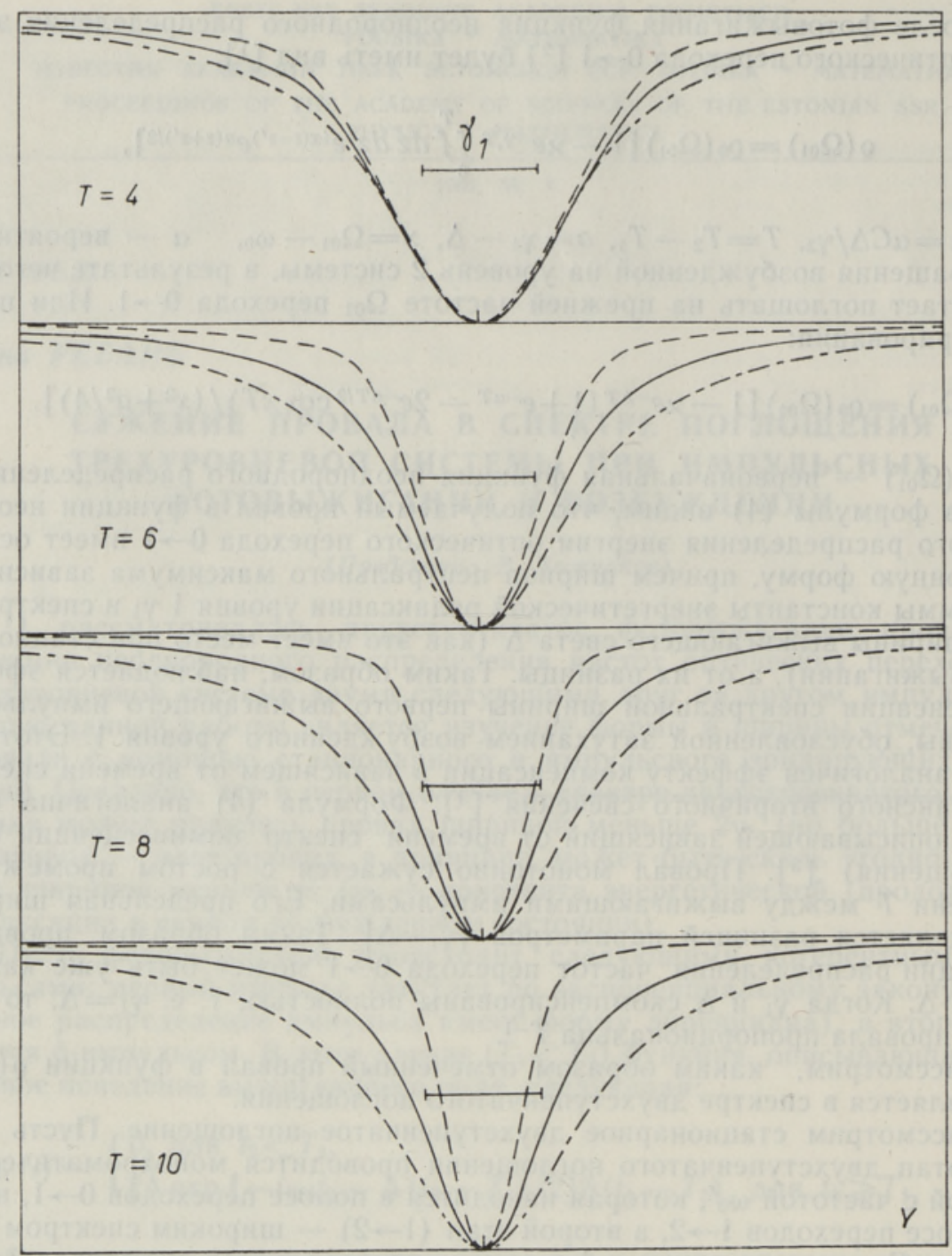

Сужение провала в спектре двухступенчатого поглощения при возрастании промежутка времени $T$ между выжигающими импульсамн. Стационарное возбуждение (зондирование) - сплошная линия и импульсное возбуждение (зондирование) такими же импульсами как и при выжигании - штриховая линия $\left(\Delta^{\prime}=\Delta, T^{\prime}=T\right)$.

Спектральная ширина первого импульса $\Delta=0,99 \gamma_{1}$.

Штрих-пунктирной линией отмечен спектральный провал в спектре двухступенчатого поглощения при стационарном выжигании и возбуждении монохроматическими волнами (его ширина $2 \gamma_{1}$ ). Промежуток времени $T$ дан в $\gamma_{1}^{-1}$.

где

$$
\begin{gathered}
y=\omega_{0}^{\prime}-\omega_{0}, \\
\beta_{1}=y^{2}+\frac{\left(\gamma_{1}+a\right)^{2}}{4}=y^{2}+\frac{\left(2 \gamma_{1}-\Delta\right)^{2}}{4}, \\
\beta_{2}=y^{2}+\frac{\left(\gamma_{1}-a\right)^{2}}{4}=y^{2}+\frac{\Delta^{2}}{4} .
\end{gathered}
$$

Таким образом, провал в функции неоднородного распределения, имеющий форму (4), приводит также к провалу в спектре двухступенчатого 
поглощения. С ростом времени $T$ между выжигающими импульсами ширина провала, наблюдаемая в отмеченном эксперименте, уменьшается (см. рисунок). Минимальная ширина провала в спектре (6) получается при $\gamma_{1}=\Delta$. В этом случае с ростом $T$ шмрина провала в спектре стремится к ширине, обусловленной затуханием возбужденного уровня $1-\gamma_{1}$. При $a=10^{-2} \gamma_{1}$, начиная с $T>4 \gamma_{1}^{-1}$, ширина провала меньше минимально возможной ширины провала в условиях стационарного выжигания провала - $2 \gamma_{1}$ (при выжигании монохроматической волной частоты $\left.\omega_{0}\right)$.

Поглощение в максимуме провала $(y=0)$ при $a T \ll 1$ :

$$
I_{s}(0) \sim 1-4 \varkappa e^{-\Delta T}\left[\gamma_{1} T+2 e^{-\gamma_{1} T / 2}-2\right] .
$$

Провал с максимальной глубиной получаем при $T=2 \gamma_{1}^{-1}$, тогда $I_{s}(0) \sim$ $\sim 1-0,4 \varkappa$. Отметим, что при $T=4 \gamma_{1}^{-1}$ глубина провала $\sim 42 \%$, при $T=6 \gamma_{1}^{-1}-\sim 10 \%$, при $T=8 \gamma_{1}^{-1}-\sim 2 \%$ и при $T=10 \gamma_{1}^{-1}-\sim 0,4 \%$ от максимальной глубины провала.

2. Рассмотрим двухступенчатое поглощение двух световых импульсов, содержащих резонансные с соответствующими переходами частоты (первый импульс в резонансе с переходом $0 \rightarrow 1$, но не с $1 \rightarrow 2$, второй в резонансе только с переходом $1 \rightarrow 2$ ). В общем случае вероятность найти систему в момент времени $t$ в состоянии 2 следующая:

$$
\begin{gathered}
W(t)=\int d \Omega_{01} \varrho\left(\Omega_{01}\right) \int_{-\infty}^{t} d t_{1} d t^{\prime}{ }_{1} \int_{-\infty}^{t_{1}} d t_{2} \int_{-\infty}^{t_{1}^{\prime}} d t^{\prime}{ }_{2} f\left(t_{2}, t_{1}\right) f^{*}\left(t_{2}^{\prime}, t_{1}^{\prime}\right) \times \\
\times F\left(t_{1}^{\prime}-t_{1}, t-t_{1}, t_{1}^{\prime}-t_{2}^{\prime}, t_{1}-t_{2}\right),
\end{gathered}
$$

где функция $f\left(x_{1}, x_{2}\right)$ описывает временное поведение поглощаемого поля.

Пусть поглощаемые импульсы будут такого же типа, как и импульсы, используемые при фотовыжигании, - первый импульс является когерентным и затухает по экспоненциальному закону, а второй является когерентным $\delta$-импульсом. Тогда $\left(T_{1}{ }^{\prime \prime}<T_{2}{ }^{\prime}\right)$

$$
f\left(t_{2}, t_{1}\right)=\left\{\begin{array}{l}
0, \quad \text { для } t_{2}<T_{1}^{\prime}, \\
\sqrt{\Delta^{\prime}} e^{-i \omega_{0}^{\prime} t_{2}-\Delta^{\prime}\left(t_{2}-T^{\prime}{ }_{1}\right) / 2} \delta\left(t_{1}-T^{\prime}{ }_{2}\right), \quad \text { для } t_{2} \geqslant T_{1}^{\prime},
\end{array}\right.
$$

где $T_{1}{ }^{\prime}-$ момент времени прохождения через центр системы максимума первого импульса, $T_{2}{ }^{\prime}-$ момент времени прохождения через центр системы второго импульса, $\omega_{0}^{\prime}$ - частота максимума и $\Delta^{\prime}$ спектральная ширина первого импульса, $\left(\Delta^{\prime}\right)^{-1}-$ его длительность во времени.

В этом случае двухступенчатое поглощение $I_{i}$ пропорционально вероятности найти снстему в состоянии 2 в момент времени $T_{2}^{\prime}: I_{i} \sim$ $\sim W_{i}\left(T_{2}^{\prime}\right)$.

Подставляя формулы (2), (3) и (9) в формулу (8) и предполагая, что $\varrho_{0}\left(\Omega_{01}\right)=\varrho_{0}=$ const, после интегрирования формулы (8) получаем спектр двухступенчатого поглощения:

$$
\begin{gathered}
I_{i}(y) \sim 2 \pi \varrho_{0} C \Delta^{\prime} e^{-\Delta^{\prime} T^{\prime}}\left\{\frac{1}{b}\left(1-e^{-b T^{\prime}}\right)-\right. \\
-x e^{-\Delta T}\left[\frac{(a+b)}{a b \delta_{1}}\left(1-e^{-a T-b T^{\prime}}\right)+\frac{(a-b)}{a b \delta_{2}}\left(e^{-a T}-e^{-b T^{\prime}}\right)-\right.
\end{gathered}
$$




$$
\begin{gathered}
-\frac{2}{\delta_{1} \delta_{2}} \times\left\{\begin{array}{r}
e^{-(a+b) T^{\prime} / 2}\left(\frac{1}{a}\left(y^{2}+\frac{\left(b^{2}-a^{2}\right)}{4}\right)\left(1-e^{-a\left(T-T^{\prime}\right)}\right) \cos y T^{\prime}+\right. \\
\left.\left.\left.+\left(1+e^{-a\left(T-T^{\prime}\right)}\right) y \sin y T^{\prime}\right)\right]\right\}, \text { при } T^{\prime}<T, \\
e^{-(a+b) T / 2}\left(\frac{1}{b}\left(y^{2}+\frac{\left(a^{2}-b^{2}\right)}{4}\right)\left(1-e^{-b\left(T^{\prime}-T\right)}\right) \cos y T+\right.
\end{array}\right. \\
\left.\left.\left.+\left(1+e^{-b\left(T^{\prime}-T\right)}\right) y \sin y T\right)\right]\right\}, \text { при } T<T^{\prime},
\end{gathered}
$$

где $b=\gamma_{1}-\Delta^{\prime}, T^{\prime}=T^{\prime}{ }_{2}-T^{\prime}{ }_{1}$,

$$
\begin{aligned}
& \delta_{1}=y^{2}+\frac{(a+b)^{2}}{4}=y^{2}+\frac{\left(2 \gamma_{1}-\Delta-\Delta^{\prime}\right)^{2}}{4}, \\
& \delta_{2}=y^{2}+\frac{(a-b)^{2}}{4}=y^{2}+\frac{\left(\Delta-\Delta^{\prime}\right)^{2}}{4} .
\end{aligned}
$$

В случае, когда обе пары импульсов одинаковые, т. е. $\Delta=\Delta^{\prime}$ и $T=T^{\prime}$, спектр поглощения следующий:

$$
\begin{gathered}
I_{i}(y) \sim 2 \pi \varrho_{0} C \Delta e^{-\Delta T}\left\{\frac{1}{a}\left(1-e^{-a T}\right)-\right. \\
\left.-\frac{x}{\delta} e^{-\Delta T}\left[\frac{1}{a}\left(1-e^{-2 a T}\right)-2 e^{-a T} \frac{\sin y T}{y}\right]\right\},
\end{gathered}
$$

где $\delta=y^{2}+a^{2}$.

Из формул (10) и (11) (см. также рисунок) видим, что в случае рассматриваемого поглощения двух импульсов также имеет место эффект компенсации - компенсируются ширина, обусловленная затуханием возбужденного уровня $1\left(\gamma_{1}\right)$, и спектральная ширина первого поглощаемого импульса $\left(\Delta^{\prime}\right)$. С ростом промежутка времени между возбуждающими импульсами ширина провала уменьшается. Она минимальна при $\Delta=\Delta^{\prime}=\gamma_{1}$ (полный эффект компенсации). Таким образом, используя рассматриваемое импульсное выжигание провала и импульсное двухступенчатое поглощение, можно, в принципе, получить сколь угодно узкие провалы в спектре двухступенчатого поглощения, т. е. шириной не только меньше $2 \gamma_{1}$, но и меньше $\gamma_{1}$. Например, в случае $a=b=10^{-2} \gamma_{1}$ и $T=T^{\prime}$ (см. рисунок) при $T=4 \gamma_{1}^{-1}$ ширина провала $\sim 1,9 \gamma_{1}$, при $T=6 \gamma_{1}^{-1}$ - $\sim 1,2 \gamma_{1}$, а при $T=8 \gamma_{1}^{-1}$ ширина провала меньше $\gamma_{1}: \sim 0,9 \gamma_{1}$; далее, при $T=10 \gamma_{1}^{-1}$ ширина провала в спектре $\sim 0,7 \gamma_{1}$.

Поглощение в максимуме провала $(y=0)$ при $a T \ll 1$ (случай $a=b$; $\left.T=T^{\prime}\right)$

$$
I_{i}(0) \sim\left(\gamma_{1} T\right) e^{-\gamma_{1} T}-\frac{2 \varkappa}{3}\left(\gamma_{1} T\right)^{3} e^{-2 \gamma_{1} T} .
$$

Величина начального уровня $I_{0} \sim \gamma_{1} T e^{-\gamma_{1} T}$ зависит от промежутка времени $T$ между импульсами. Отношение глубины провала $\Delta I \sim \frac{2 x}{3}\left(\gamma_{1} T\right)^{3} \exp \left(-2 \gamma_{1} T\right) \quad$ относительно шума

$$
f(T)=\frac{\Delta I}{\sqrt{I_{0}}}=\frac{2 \varkappa}{3}\left(\gamma_{1} T\right)^{2,5} e^{-1,5 \gamma_{1} T}
$$

максимальное при $T=1,67 \gamma_{1}^{-1}: f_{\max } \sim 0,2 x$. 
Оิтметим, что при $T=4 \gamma_{1}^{-1}$ функция $f(T)$ составляет $\sim 27 \%$, при $\bar{T}=$ $=6 \gamma_{1}^{-1}-\sim 4 \%$, при $T=8 \gamma_{1}^{-1}-\sim 0,4 \%$ и при $T=10 \gamma_{1}^{-1}-0,03 \%$ от $f_{\max }$.

В данном рассмотрении не учтена фазовая (поперечная) релаксация возбужденных уровней. Учет фазовой релаксации первого возбужденного уровня приводит как к уширению провала в функции неоднородного распределения энергии оптического перехода $0 \rightarrow 1$, так и к дополнительному уширению при регистрации провала в спектре двухступенчатого поглощения в обоих рассмотренных случаях регистрации.

Компенсируется только ширина, обусловленная энергетической релаксацией уровня 1. Она компенсируется спектральной шириной первого выжигающего импульса $\Delta$ (при выжигании) и спектральной шириной первого поглощаемого импульса $\Delta^{\prime}$ (при импульсном поглощении).

Автор благодарен К. К. Ребане, В. В. Хижнякову и Я. Кикасу за обсуждение работы.

\section{ЛИТ Е РА Т У РА}

1. Ребане И. Изв. АН ЭССР. Физ. Матем., 35, № 3, 296-301 (1986).

2. Rebane, L. A., Gorokhouskii, A. A., Kikas, J. V. Appl. Phys. B, 29, 235-250 (1982).

3. Хижняков В. В., Ребане И. К. Ж. эксперим. и теор. физ., 74, вып. 3, 885-896 (1978); 77, вып. 4, 1302-1312 (1979).
Институт физики
Академии наук Эстонской ССР
Поступила в редакцию
$30 / \mathrm{I} \quad 1986$

\section{Inna REBANE}

\section{AUGU KITSENEMINE KOLMENIVOOLISE SUSTEEMI NEELDUMISSPEKTRIS IMPULSS-FOTOPOLETAMISEL JA -ERGASTAMISEL}

Auk kaheastmelisel fotopõletamisel optiliste üleminekute sageduste mittehomogeensesse jaotusfunktsiooni kahe üksteisele järgneva valgusimpulsiga juhul, kui esimeseks impulsiks on koherentne eksponentsiaalselt kustuv impulss ja teiseks $\delta$-impulss, võib olla kitsam statsionaarses režiimis pōletamisel saadavast august. On uuritud selle augu kuju ja laiust, kasutades sondeerimiseks nii statsionaarset valgust kui ka valgusimpulsse. Kaheastmelises neeldumisspektris on esimesel juhul võimalik saada auk, mis on kitsam kui $2 \gamma_{1}$, kuid laiem kui $\gamma_{1}\left(\gamma_{1}\right.$ - esimese ergastatud oleku energeetilise relaksatsiooni konstant). Teisel juhul, kui sondeerimisel kasutada sama impulsside paari nagu augu pôletamiselgi, vōib auk kaheastmelises neeldumisspektris olla ka kitsam kui $\gamma_{1}$.

\section{Inna REBANE}

\section{NARROWING OF THE HOLE IN THE ABSORPTION SPECTRUM OF A THREE- LEVEL SYSTEM ON PULSED PHOTOBURNING AND EXCITATION}

On the two-step photoburning of a hole in the function of the inhomogeneous distribution of optical transition frequencies in a three-level system by two successive light pulses, in the case of an exponentially decaying first coherent pulse and the second $\delta$-pulse, the hole can be narrower than the one obtained in stationary burning conditions. The shape and width of the hole has been studied by means of stationary and pulsed probing radiation. It is shown that in the former case, a hole of the width less than $2 \gamma_{1}$ but larger than $\gamma_{1}$ ( $\gamma_{1}$ is the constant of energetic (longitudinal) relaxation in the first excited state), can be ubtained in the two-step absorption spectrum. In the latter case, if on probing a pair of pulses similar to the one applied on burning is used, the hole in the two-step absorption spectrum can, in principle, be also of the width less than $\gamma_{1}$. 